\title{
Research on Dispatching Management of High Proportion New Energy Grid in the Electricity Market Environment
}

\author{
Bolin Zhang ${ }^{1,}$, , Chong Shao ${ }^{1}$, Chunxiang Yang ${ }^{1}$, Xiaobin Zhang ${ }^{1}$, \\ Tianyu Zhang ${ }^{2, b}$, and Yuguo Chen ${ }^{2}$ \\ 1. State Grid Gansu Electric Power Company, Lanzhou 730030, China \\ 2. Beijing Tsintergy Technology Co., Ltd., Beijing 100084, China \\ a bolin.zhang@sgcc.com.cn, ${ }^{b}$ tianyu8718@163.com
}

\begin{abstract}
The electricity spot market can reflect the power supply and demand situation in real time through price signals, guiding users to shift peaks and fill valleys, and enhancing the level of new energy consumption. Gansu Province, one of the first pilot spot market provinces in China, has designed a bilateral spot market for generations and users. The power supply mode and grid operation mode will undergo changes according to the operation results of the power market to achieve the optimal allocation of power resources, thus posing a huge challenge to the original grid dispatching mode. The article analyses the dispatch management articulation model based on the high penetration of new energy grids in the power market reform, adapts the work focus after changes in the power market business, and explores efficient dispatching business processes for the power spot market to ensure a smooth transition of dispatching work and effective integration of the market business.
\end{abstract}

Keywords: Electricity Spot Market; New Energy; Dispatch Management; Linking-up Mode.

\section{Introduction}

By the end of 2020, the total installed capacity of wind power on the grid nationwide reached 281.53 million kilowatts, accounting for $12.8 \%$ of the total installed capacity of the national power supply and $28.6 \%$ of the installed capacity of non-fossil power sources [1]. Alongside the rapid development of renewable energy, there has always been tremendous pressure to ensure the utilization rate of renewable energy generation equipment and to reduce abandoned wind power and photovoltaic. The new stage of China's power system reform and spot market construction pilot will provide a new approach to the market-based large-scale consumption of new energy [2]. Gansu Province is in the main area of renewable energy generation, with a rapid development of wind and solar power industries. By the end of June 2021, the installed capacity of wind power in Gansu reached 13,121,900 kilowatts and photovoltaic power generation reached 9,251,100 kilowatts, with the installed capacity of renewable energy reaching $42.3 \%$, of those new energy installations overtaking thermal power as the first major energy source in the provincial power system. However, Gansu is also one of the most serious regions suffering power abandonment owing to the constraints of limited local consumption space, cross-regional transmission channels and insufficient peaking capacity. As one of the first pilot provinces of the national spot market, Gansu Province is also actively exploring a spot market system to promote the consumption of new energy, establishing a spot market mechanism for renewable energy penetration and launching a trial run of the spot market at the end of 2018, and so far Gansu has completed five settlement trial runs.

The Gansu spot market has gone through two stages, with the market initially adopting a unilateral centralized competition model on the power generation side, where the market players include thermal power units, hydropower units and renewable energy farms [3]. As market awareness on the power generation side is gradually cultivated, Gansu spot market entered the second stage in 2021, introducing user-side participation in power spot trading to achieve effective transmission of spot price signals to the user side and further release the price elasticity of power demand. The advantage of low marginal cost of renewable energy will help to direct "load following source" and expand the space for energy consumption. 
It is difficult for the traditional power dispatching system to adapt into the Market-oriented reform, and new requirements have been put forward to adjust the departmental structure and priority of work in response to the changes in business. This paper presents the challenges of the market-based environment in which the high penetration of new energy is consumed, also proposes a dispatch management transition method for the background of Market-oriented reform, explores efficient Market-oriented dispatch business processes, ensuring a smooth transition of dispatch tasks and effective integration of market business, to provide guidance for the dispatch management business of other high penetration new energy grids in a Market-oriented environment.

\section{Gansu Electricity Spot Market Development Scheme}

The Gansu spot market is carried out in a centralized bidding mode with full power in day-ahead and real-time, and renewable energy sources trade directly in the provincial spot market via volume quotations. Considering the current mechanisms and policies for guaranteeing the consumption of new energy, new energy concessions and pro-poor units are involved in the spot market as price takers in the day-ahead market, which means that only the 96-point output forecast curve of the following day is declared, and priority is given to clearing and consumption in the spot market. The remaining new energy sites will be bided centrally with thermal power units, aiming to maximize social welfare and clear the winning power curve unifiedly. If there still exists abandoned wind power and photoelectricity in the provincial spot market after clearing, new energy sites can compete in crossregional inter-provincial surplus renewable energy power trading.

The objective function of spot market bid-offer clearing mathematical models is to maximize social welfare:

$$
\min \left(\sum_{i=1}^{N} \sum_{t=1}^{T}\left[C_{i, t}\left(P_{i, t}\right)+C_{i, t}^{U}\right]-\sum_{u=1}^{M} \sum_{t=1}^{T}\left(C_{u, t}\left(L_{u, t}\right)\right)+\sum_{s=1}^{N S} \sum_{t=1}^{T} K\left[S L_{s}^{+}+S L_{s}^{-}\right]\right)
$$

where $T$ is the total number of periods, where one period every 15 minutes on $D$ day and 96 periods are considered; $N$ is the total number of units; and $M$ is the number of users participating in the dayahead spot market quoted; $P_{i, t}$ is the output of unit $i$ at time $t ; L_{u, t}$ is the electricity consumption of market user $u$ at time $t ; C_{i, t}\left(P_{i, t}\right), C_{i, t}^{u}$ are the operating cost and start-up cost of unit $i$ at time $t$ respectively; $C_{u, t}\left(L_{u, t}\right)$ is the segmented offer curve of market users. $K$ is the power flow of the constrained relaxation penalty factor used for market clearing optimization; $S L^{+}{ }_{S}, S L_{S}{ }_{S}$ are the forward and reverse flow relaxation variables for section s respectively; $N S$ is the total number of sections.

\section{Impact of the Electricity Market on Traditional Grid Dispatching Methods}

Under the market mechanism, grid dispatching agency will perform the following functions: realtime generation unit dispatching and control, analysis of the grid operation modes, arrangement of unit commitment, liaison line plans, generation and transmission device maintenance plans, balanced execution of power plans, renewable energy consumption and security checks for medium and longterm transactions, etc. It will also include new functions, covering the formulation of rules for electricity spot market transactions, the construction of spot market operation platform, the organisation of spot transactions, the execution of plans and the disclosure of information under the rules.

Before market reform, the traditional dispatching method was based on the planned electricity output of each power plant according to the average utilization hours within the same provincial grid. However, lack of consideration on the plant and unit performance may lead to the out-of-order development on the higher polluting and inefficient small thermal power plants, small cogeneration plants and oil-fired power plants. Moreover, the economics of the generation side as well as the pollution to the environment are becoming more serious, which is against the national target of 
"Carbon peak and neutrality ", therefore it is needed to analyse the direction of modification of the traditional dispatch method.

The grid dispatch management method will directly affect the reliability, stability and security of the electricity supply of the power system. The market-based reforms will affect the traditional power dispatch management business in the following ways.

\subsection{Power Generation Plans}

The non-Market-oriented generation plan is mainly established by the dispatching agency based on annual and monthly priority generation hours and medium and long-term power purchase and sale contracts, based on load forecasts, inter-provincial liaison line plans, unit and line maintenance plans, and in accordance with the progress of each unit's base power and medium and long-term monthly plans, to set unit start and stop plans as well as daily operational output plans.

Under a Market-oriented system, such generation plan forming mechanisms will be broken. the generation capacity of each power plant is given from market bids, especially with the implementation of the spot market, the generation curves of power plants will be shaped by market bidding. The forming patterns change the conventional grid operation mode. As the grid starts to deviate from the equilibrium operation point, it gradually approaches the safety boundary driven by market-based trading.

\subsection{Safety Checks}

When there was no spot market, medium and long-term power transactions needed to be physically executed, so the security checks of them were the core of conventional dispatch management operations. The medium and long-term security check is based on safe operation, with annual power balance as the target, and rolling adjustment plans so as to guarantee the security margin of grid operation and reserve the necessary adjustment space for dispatch.

One of the key demands of Market-oriented reform is to construct a spot market for electricity. Time-wise, the medium and long-term security checks need to be refined to day-ahead security checks, and should be complemented with intelligent security checking mechanisms and tools that include day-ahead and intra-day full cycles.

\subsection{Supply and Demand Balance}

In the conventional standby management method, the scheduling of the standby is command-based, i.e. a certain number of standbys are arranged or designated in each belonging plant under defined operating conditions, and in a breakdown situation, a response order is issued by system scheduling. However, in a market environment where power plants are separate economic entities, standby services should be considered essentially a commodity and their use should be financially compensated. The traditional compulsory access to back-up services will no longer apply.

\section{Design of Dispatch Management Mechanism for High Penetration Renewable Energy Grids}

In an electricity market environment, the original process of balancing the system will change significantly, with the objective still being to ensure real-time balancing of the system, but the methods of choosing frequency regulation and reserved units will change, from a selection based mainly on manual experience to a strict implementation based on market rules and predetermined processes.

The spot market runs uninterruptedly, and generation plans are rolled over by the spot system every 15 minutes, with each subsequent two-hour rollover. Dispatchers need to handle all types of planned and unplanned events in a timely manner and pass these changes to the real-time market clearing system model immediately to ensure that the real-time market optimization results are in line with the latest grid operation. From the real-time dimension, the market implements real-time 
economic optimal dispatch by performing full power optimization at the operating time point (typically 15 minutes before the real-time operating point), which is an important tool for real-time balancing of the system.

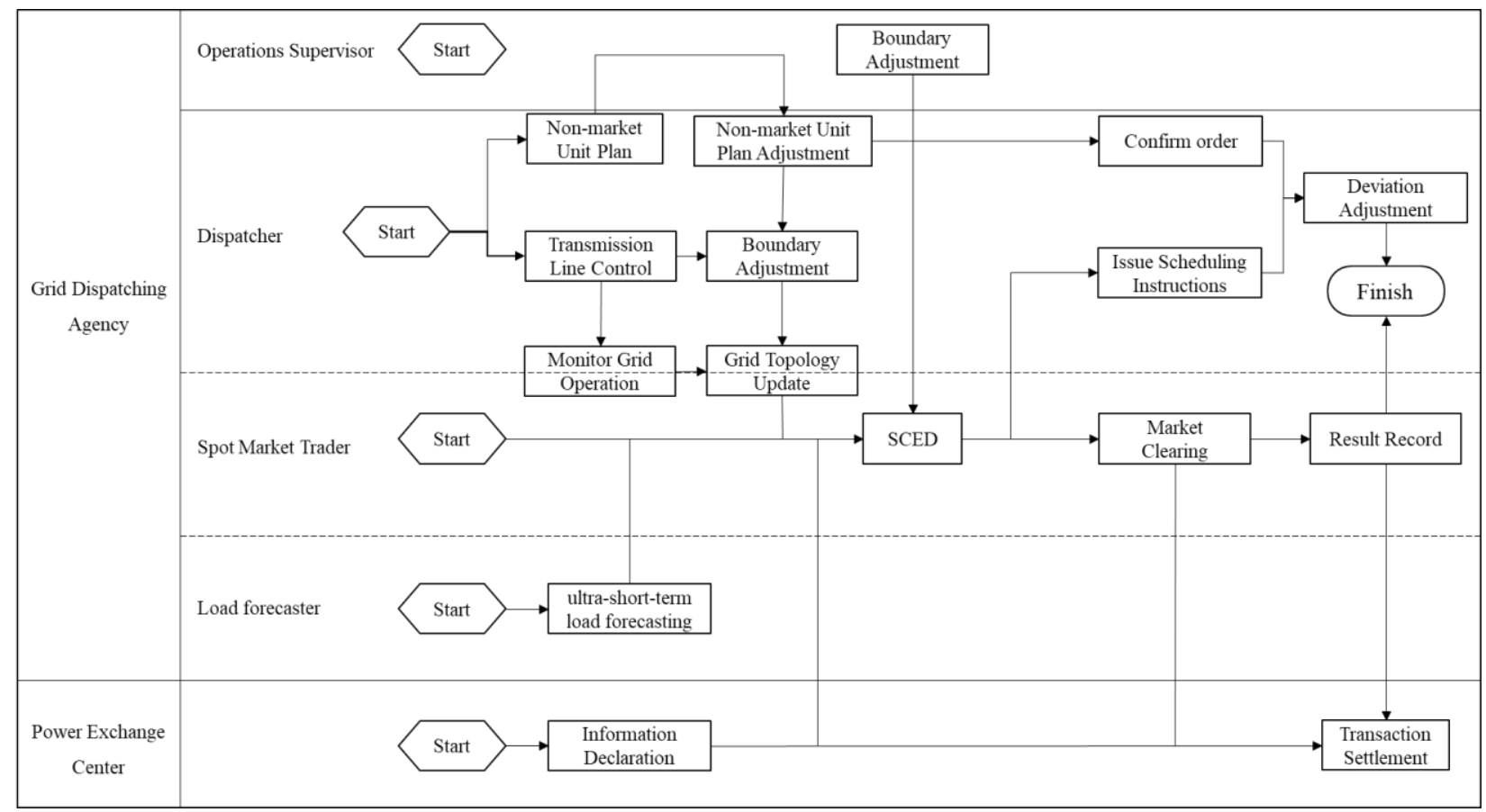

Fig 1. Spot market flow

In the organization of the spot market, dispatch management can be carried out in the following manners.

The dispatch and operation supervisor are responsible for reviewing the next day's generation plan for non-market units, with emphasis on checking generation and transmission plans, maintenance arrangements and section control requirements, and ensuring that there are no problems before performing sign-off procedures.

According to the real-time dispatching operation of the grid, the impact of the operation changes on the real-time market SCED should be analyzed, and the internal parameters and boundary conditions of the algorithm should be adjusted to meet the requirements of the SCED, thus making the optimization algorithm model consistent with the current grid operation. When changes in grid operation occur, which includes the definite adjustment on the spot market for inter-provincial transmission and reception, abnormal disposal and accident handling. Changes in grid operation and dispatch requirements will be transmitted to real-time optimization through automatic transmission from the D5000 or manual manipulation of the spot system.

Real-time market and economic dispatch optimization are carried out 15 minutes in advance and automatic adjustment orders are issued once completed. However, due to deviations in load forecasts and changes in demand, there still exists an imbalance between system supply and demand at the realtime operating nodes, which needs to be balanced through frequency regulation or standby supplied by the ancillary services market. The on-duty dispatcher implements operational regulation of the grid in accordance with the daily generation plan and makes dispositions for emergency shutdowns, grid faults, incident handling, maintenance extensions or changes, and analyses the impact on the real-time market SCED optimization algorithm boundary based on the disposition results.

\section{Design of Market-oriented Multi-level Dispatch Management Process}

In the power market environment, the daily generation plan is mainly determined by the relevant clearing algorithm in the spot market, and the dispatching of the former daily generation plan-making 
task mainly turns into the determination of market clearing boundary conditions. However, the model for optimising generation plans on a day-ahead basis will require the pooling of information on dispatching arrangements and planned output at all levels, and the business process for day-ahead generation plans involves dispatching at all levels across the grid. The main framework of the national unified power market is a "unified market with two levels of operation". The unified market represents a unified market framework, unified core rules, unified operating platform and unified service specifications to support the optimal allocation of energy resources on a national scale. The two levels of operation represent the division of the unified market into two levels of operation: inter-provincial and intra-provincial trading, with an effective interface between the two levels of trading operations.

Recent years, with the construction and development of China's ultra-high voltage power grids, the unified and coordinated operation of power grids at all levels has gradually formed, and the national power grid has presented a new grid model, with the grid gradually serving as the main platform for the optimal allocation of energy resources, and the features of coordinated operation of power grids across all levels being apparent. The conventional dispatching mode of China's power grids, which is dominated by self-balancing, will gradually collapse, and it is urgent to transform into a Multi-level coordinated dispatching mode with provincial power grids as a unit and expand to regional and national power grids, so as to comprehensively improve the efficiency of the unified and coordinated operation of large-scale power grids and fully play a vital role in the rational and effective distribution of energy resources by large power grids.

To achieve the national "double carbon" target, renewable energy generation will gradually become the main source of energy for the power grid. While bringing energy saving and environmental benefits to the grid, its intermittent and fluctuating power output poses a serious challenge to the grid's short-term power balance and real-time safe operation. It is difficult to meet the demand for safe and economic operation when a large number of new energy sources are connected to the provincial grid. Instead, it is crucial to rely on the Multi-level unified and coordinated operation of the grid and to reserve the operating space for hydropower and wind power in advance, so as to greatly improve the capacity of renewable energy consumption and give full play to the benefits of renewable energy in the overall scope.

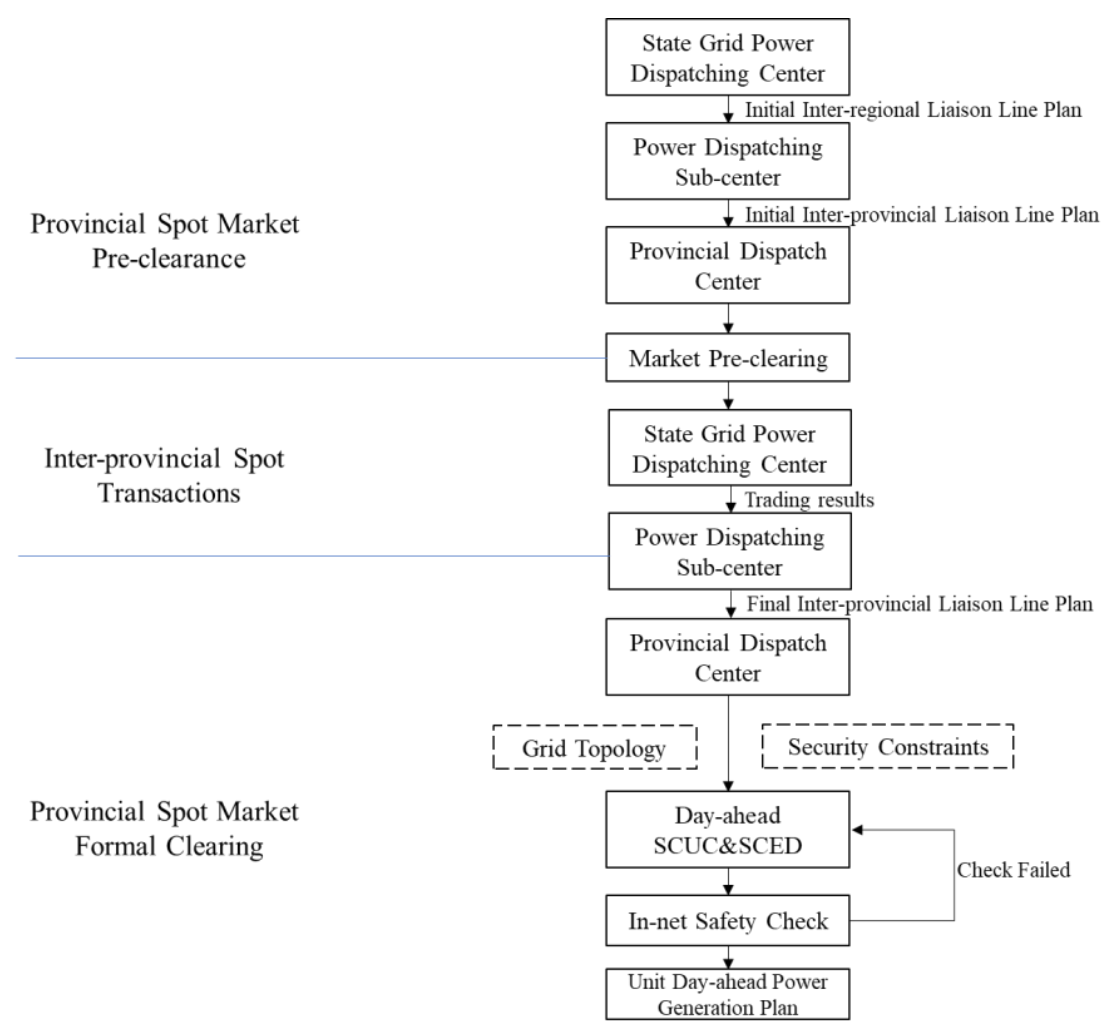

Fig 2. The process of linking the Multi-level dispatch and management methods of the power grid 
Intra-provincial electricity spot trading (pre-clearance). The Sub-center conducts safety checks on the whole grid according to the preliminary inter-regional liaison line plan issued by the State Grid Power Dispatching Center, and formulates the preliminary inter-provincial liaison line plan to be issued to the provincial dispatching center. Each provincial dispatching center is responsible for aggregating and processing the relevant information uploaded by the local coordinators and the medium and long-term physical contracts within the province, together with the preliminary interprovincial liaison line plan for the pre-clearance of the provincial spot market.

Inter-provincial spot transactions. The provincial dispatchers determine the provincial power supply gap and report it to the sub-center based on the preclearance results of the provincial dayahead market, combined with load forecasts, while the sub-center organizes the four provinces to participate in inter-provincial spot trading. The national dispatch center delivers the preliminary interprovincial spot trading results to the sub-center, who carries out the main network reliability checks especially for the inter-provincial cross-sections. Based on the results of inter-provincial spot trading clearances, the national dispatch center forms the final inter-regional liaison line plan and issues it to the sub-center.

Provincial spot market formal clearance. The provincial dispatchers adjust the provincial spot market clearing boundary by considering the topology and security constraints of the whole grid, and carries out day-ahead security constraint unit commitment (SCUC) and security constraint economic dispatch optimization, which will then formulate the network day-ahead generation plan by the Subcenter.

\section{Summary}

Taking Gansu Province as an example, this paper introduces the construction plan of the electricity spot market based on high proportion of renewable energy, and analyzes the improprieties of the traditional dispatch mode from the perspective of market form. Combined with the relevant policies of the electricity market, a dispatch management mechanism is designed, and an overall dispatch management process is designed in consideration of the Multi-level market. This paper processes a new management model for dispatch centers in different provinces, which can promote the efficiency of dispatch operations under Market-oriented conditions.

\section{References}

[1] National Bureau of Statistics. China Electricity Development Report 2020 [N]. People's Daily,2021-0719.

[2] WANG Caixia, LEI Xuejiao, LIU Lihua, et al. Design of Short-term Renewable Energy Integration Mechanism in the Electricity Market Transition Period[J]. Journal of Global Energy Interconnection, 2018,1(05):565-573.

[3] Wang Chunwei. Research on Coping Strategies of Gansu New Energy Spot Market[J]. Energy Conservation \& Environmental Protection, 2019(04):84-85.

[4] Wang Xiaohai, Qi Jun, Jiang Xiwei, Liu Yiyang, Xu Fei, Li Wei. Research on the design and trading mechanism of flexibility products in the electricity market[J]. China Market,2020(05):65-68.

[5] WANG Ke, YAO Jianguo, YAO Liangzhong, et al. Survey of Research on Flexible Loads Scheduling Technologies[J]. Automation of Electric Power Systems, 2014,38(20):127-135.

[6] He Guixiong, Cao Nianlin, Jiang Limin, et al. Research on peak-load regulating with participation of high-use industrial consumers in wind power rich area[J]. Renewable Energy Resources, 2015, 33 (04): 491-496.

[7] BAI Xuexiang. Research on Application of Demand Response Resources in Smart Power System and its Benefit Evaluation[D]. North China Electric Power University,2017.

[8] Yao Chunxiao. A Study of Identification Method of Wide-area Adjustable Load[D]. North China Electric Power University (Beijing),2019. 
[9] ZOU Peng, CHEN Qixin, XIA Qing, et al. Logical Analysis of Electricity Spot Market Design in Foreign Countries and Enlightenment and Policy Suggestions for China[J]. Automation of Electric Power Systems.

[10] XUE Xiaocen, WU Qiaoling, ZHANG Jiguang. Experience of Foreign Electricity Spot Market and Discussion of Spot Market Construction in China[C]. Power Market Committee of China Electrical Engineering Society, 2018:62-67.

[11] MA Hui, CHEN Yuguo, CHEN Ye, et al. Mechanism Design of Southern China (Starting from Guangdong Province) Electric Spot Market[J]. Southern Power System Technology, 2018,12(12):42-48.

[12] LIANG Zhifei, CHEN Wei, ZHANG Zhixiang, et al. Discussion on pattern and path of electricity spot market design in southern region of China[J]. Automation of Electric Power Systems, 2017,41(24).

[13] XU Tanghai, et al. High Penetration of Renewable Energy Power Planning Considering Coordination of Source-load-storage Multi-type Flexible Resources[J]. Journal of Global Energy Interconnection. 